\title{
4-core partitions and class numbers
}

by

Ken Ono (Princeton, N.J.) and Lawrence Sze (University Park, Penn.)

1. Introduction. A partition of a positive integer $n$ is a non-increasing sequence of positive integers whose sum is $n$. The number of such partitions is denoted by $p(n)$. If $\Lambda=\lambda_{1} \geq \ldots \geq \lambda_{s}$ is a partition of $n$, then the Ferrers-Young diagram of $\Lambda$ is the $s$-row collection of nodes:

$$
\begin{array}{llllll}
\bullet & \bullet & \ldots & \bullet & \bullet & \lambda_{1} \text { nodes } \\
\bullet & \bullet & \ldots & \bullet & & \lambda_{2} \text { nodes } \\
\vdots & & & & & \\
\bullet & \ldots & \bullet & & \lambda_{s} \text { nodes }
\end{array}
$$

Label the nodes in the Ferrers-Young diagram of a partition as if it were a matrix. Let $\lambda_{j}^{\prime}$ denote the number of nodes in column $j$. Then the hook number $H(i, j)$ of the $(i, j)$ node is defined by

$$
H(i, j):=\lambda_{i}+\lambda_{j}^{\prime}-j-i+1 .
$$

Definition 1. If $t$ is a positive integer, then a partition of $n$ is called a $t$-core of $n$ if none of the hook numbers of its associated Ferrers-Young diagram are multiples of $t$. Moreover, let $C_{t}(n)$ denote the number of $t$-core partitions of $n$.

EXAmple 1 . Let $\Lambda$ denote the partition of 9 defined by $\Lambda=5,3,1$. Then the Ferrers-Young diagram of $\Lambda$ is

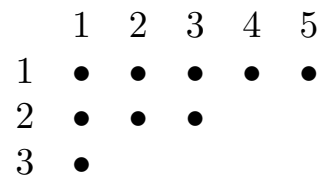

1991 Mathematics Subject Classification: Primary 11P99; Secondary 5E10, 20C20.

Key words and phrases: 4-core partitions, class numbers.

The first author is supported by NSF grants DMS-9508976 and DMS-9304580. 
The hook numbers are $H(1,1)=7, H(1,2)=5, H(1,3)=4, H(1,4)=2$, $H(1,5)=1, H(2,1)=4, H(2,2)=2, H(2,3)=1$, and $H(3,1)=1$. Therefore, if $t \notin\{1,2,4,5,7\}$, then $\Lambda$ is a $t$-core.

These partitions arise in a number of settings. In combinatorial number theory, Garvan, Kim, and Stanton [13] used them to obtain combinatorial proofs of certain special cases of the Ramanujan congruences for $p(n)$. Specifically, they proved that

$$
\begin{aligned}
& p(5 n+4) \equiv 0(\bmod 5), \quad p(7 n+5) \equiv 0(\bmod 7), \\
& p(11 n+6) \equiv 0(\bmod 11), \quad p(25 n+24) \equiv 0(\bmod 25),
\end{aligned}
$$

by defining explicit statistics, called cranks, which divide the relevant sets of partitions into equinumerous classes.

In representation theory, $t$-cores for $t$ prime first arose in connection with Nakayama's conjecture $[21,33]$ which describes the distribution of characters of the symmetric group into Brauer blocks (see [6, 20, 21, 33] for definitions). Brauer blocks describe the interplay between irreducible complex characters and the irreducible $p$-modular characters over finite fields with characteristic $p$. More recently, Fong and Srinivasan [11] showed how these partitions arise again in a similar context. They proved that $t$-cores, even when $t$ is composite, describe the distribution of characters of finite general linear groups and unitary groups into Brauer blocks. Of course, partitions arise in a number of other important ways in representation theory. For an important recent development see [2].

As a consequence, the arithmetic properties of $C_{t}(n)$, the number of $t$ core partitions of $n$ has been of interest in combinatorial number theory and representation theory. Hence $C_{t}(n)$ has been the focus of recent investigation. For instance, Garvan [12] proved some "Ramanujan-type" congruences for $C_{p}(n)$ for certain special small primes $p$, and Hirschhorn and Sellers [18] proved the following multiplicative formulas for $C_{4}(n)$ :

$$
\begin{aligned}
C_{4}\left(3^{2 \lambda+1} n+\frac{5 \cdot 3^{2 \lambda}-5}{8}\right) & =3^{\lambda} C_{4}(3 n), \\
C_{4}\left(3^{2 \lambda+1} n+\frac{13 \cdot 3^{2 \lambda}-5}{8}\right) & =\left(2 \cdot 3^{\lambda}-1\right) C_{4}(3 n+1), \\
C_{4}\left(3^{2 \lambda+2} n+\frac{7 \cdot 3^{2 \lambda+1}-5}{8}\right) & =\frac{3^{\lambda+1}-1}{2} C_{4}(9 n+2), \\
C_{4}\left(3^{2 \lambda+2} n+\frac{23 \cdot 3^{2 \lambda+1}-5}{8}\right) & =\frac{3^{\lambda+1}-1}{2} C_{4}(9 n+8),
\end{aligned}
$$

where $\lambda$ is a positive integer. They also conjectured similar multiplicative properties for $C_{4}(n)$ for other primes $p$. In this paper, we prove these conjectures using the index formulae for class numbers. 
The $t$-core conjecture has been the topic of a number of papers $[10,12$, $16,23,24,25,29,30]$. This conjecture asserted that if $t \geq 4$, then $C_{t}(n)>0$ for every non-negative integer $n$. Recently, Granville and Ono $[16,29,30]$ have successfully completed the proof of this conjecture using the theory of modular forms and quadratic forms, and the proof has been generalized and simplified by Kiming [23, 24]. As a consequence of this theorem, it follows that if $p \geq 5$ is prime, then every symmetric group and every alternating group has at least one defect zero $p$-block. This resolved the last case of Brauer's problem 19 for finite simple groups, and so if $G$ is a finite simple group and $p \geq 5$ is prime, then $G$ has a $p$-defect zero character.

In this paper we examine the arithmetic properties of $C_{4}(n)$ using Gauss' theory of class numbers and the combinatorics of abaci. In representation theory, the following important fundamental result for 4-cores follows from the work of Fong and Srinivasan [11, 28]:

TheOREm 1. Let $q$ be the order of the finite field $\mathrm{GF}(q)$. If $r$ is an odd prime for which $r \mid q^{2}+1$ and $n \geq 4$, then $C_{4}(n)$ equals the number of $r$-defect zero unipotent characters of the finite general linear group $\operatorname{GL}(n, q)$.

Hence every result regarding $C_{4}(n)$ is also a statement concerning the set of suitable $r$-defect zero unipotent characters of $\mathrm{GL}(n, q)$.

2. Consequences of class number theorems. First we fix notation. Let CL $(N)$ denote the class group of discriminant $N$ binary quadratic forms, and let $h(N)$ denote its order, the discriminant $N$ class number. In this section we show that $C_{4}(n)$ is related to $h(-32 n-20)$, and then as a consequence we obtain immediate corollaries which follow from well known properties of class numbers. We follow Dirichlet's refinement in describing the composition law:

Composition LaW. Let $f, g \in \mathrm{CL}(N)$ be primitive binary quadratic forms given by

$$
f=a_{0} u^{2}+b_{0} u v+c_{0} v^{2} \quad \text { and } g=a_{1} u^{2}+b_{1} u v+c_{1} v^{2} .
$$

If $\operatorname{gcd}\left(a_{0}, a_{1},\left(b_{0}+b_{1}\right) / 2\right)=1$, then let $B$ be the unique integer $\left(\bmod 2 a_{0} a_{1}\right)$ for which

$$
B \equiv b_{0}\left(\bmod 2 a_{0}\right), \quad B \equiv b_{1}\left(\bmod 2 a_{1}\right), \quad \text { and } \quad B^{2} \equiv N\left(\bmod 4 a_{0} a_{1}\right) .
$$

Then the composition of $f$ and $g$ is defined by

$$
f \cdot g:=a_{0} a_{1} u^{2}+B u v+\frac{B^{2}-N}{4 a_{0} a_{1}} v^{2} .
$$

If $f(u, v)$ and $g(u, v)$ do not satisfy the gcd condition of the composition law, replace them by the properly equivalent forms $f(u, v)$ and $g(-v, u)$ which do satisfy the gcd condition. If $N \equiv 0(\bmod 4)$, then the form $u^{2}+$ 
$N v^{2}$ represents the identity, the principal class of $\mathrm{CL}(N)$. Furthermore, the classes containing $a u^{2}+b u v+c v^{2}$ and $a u^{2}-b u v+c v^{2}$ are inverse to each other in $\mathrm{CL}(N)$.

Returning to $t$-cores we now recall an important fact. The generating function for $C_{t}(n)[13,21,25]$ is given by the following convenient infinite product:

$$
\sum_{n=0}^{\infty} C_{t}(n) x^{n}=\prod_{n=1}^{\infty} \frac{\left(1-x^{n t}\right)^{t}}{1-x^{n}} .
$$

We use this along with the Jacobi theta function identity:

$$
\prod_{n=1}^{\infty} \frac{\left(1-x^{2 n}\right)^{2}}{1-x^{n}}=\sum_{n=0}^{\infty} x^{\left(n^{2}+n\right) / 2}
$$

to derive the following theorem for 4-cores.

THEOREM 2. If $8 n+5$ is square-free, then

$$
C_{4}(n)=\frac{1}{2} h(-32 n-20) .
$$

Pr o of. Replace $x$ by $x^{8}$ in the generating function (3), then multiply the resulting power series by $x^{5}$ to obtain the following convenient factorization using the Jacobi theta function identity:

$$
\begin{aligned}
\sum_{n=0}^{\infty} C_{4}(n) x^{8 n+5} & =x^{5} \prod_{n=1}^{\infty} \frac{\left(1-x^{32 n}\right)^{4}}{1-x^{8 n}} \\
& =x^{5} \prod_{n=1}^{\infty} \frac{\left(1-x^{16 n}\right)^{2}}{1-x^{8 n}} \prod_{n=1}^{\infty} \frac{\left(1-x^{32 n}\right)^{4}}{\left(1-x^{16 n}\right)^{2}} \\
& =\sum_{i=0}^{\infty} x^{(2 i+1)^{2}} \sum_{j=0}^{\infty} x^{2(2 j+1)^{2}} \sum_{k=0}^{\infty} x^{2(2 k+1)^{2}} \\
& =\sum_{n \geq 0} R(8 n+5, Q) x^{8 n+5} .
\end{aligned}
$$

Here $R(8 n+5, Q)$ is the number of representations of $8 n+5$ by the form $Q=x^{2}+2 y^{2}+2 z^{2}$ where $x, y$, and $z>0$ are odd. By [23, Th. 86], since the class of the form $Q$ is the sole member of its genus of ternary quadratic forms,

$$
R(8 n+5, Q)=h(-32 n-20) 2^{-t\left(d / \Omega^{2}\right)} \varrho,
$$

where $t(w)$ is the number of odd prime factors of $w, d$ is the determinant of $Q, \varrho$ depends on the discriminant, and $\Omega$ is the gcd of the two-row minor determinants of $Q$. Therefore, following Jones [22], in our case, we have $d=4$, and $\Omega=2$, also, $\varrho=\frac{1}{2}$, since $-8 n-5 \equiv-1(\bmod 4)$. Therefore, $C_{4}(n)=R(8 n+5, Q)=\frac{1}{2} h(-32 n-20)$. 
We obtain an immediate corollary which leads to an elegant formula for those $C_{4}(n)$ for which $8 n+5$ is square-free. First, we recall the definition of the Kronecker character $\chi_{D}$. Define $\chi_{D}(n)$ for positive integers $n$ by

$$
\chi_{D}(n):=\prod\left(\frac{D}{p_{i}}\right)^{a_{i}}
$$

where $n=\prod p_{i}^{a_{i}}$ is the prime factorization of $n$, and $\left(\frac{D}{p}\right)$ is the Legendre symbol when $p$ is an odd prime and

$$
\left(\frac{D}{2}\right):= \begin{cases}0 & \text { if } D \text { is even, } \\ (-1)^{\left(D^{2}-1\right) / 8} & \text { if } D \text { is odd. }\end{cases}
$$

As a consequence of the well known formula for class numbers we obtain:

COROLlaRY 1. If $n$ is a non-negative integer for which $8 n+5$ is squarefree and $D=-32 n-20$, then

$$
C_{4}(n)=-\frac{1}{64 n+40} \sum_{m=1}^{32 n+20} \chi_{D}(m) m .
$$

We immediately obtain the conjectures of Hirschhorn and Sellers.

COROllary 2. If $p$ is a prime and $N$ is a positive integer with $\operatorname{ord}_{p}(N)$ $\leq 1$, then for every positive integer $k$,

$$
C_{4}\left(\frac{N p^{2 k}-5}{8}\right)=\left(1+\frac{p^{k+1}-p}{p-1}-\frac{p^{k}-1}{p-1} \cdot\left(\frac{-N}{p}\right)\right) \cdot C_{4}\left(\frac{N-5}{8}\right)
$$

where $\left(\frac{-N}{p}\right)$ is the Legendre symbol and $\left(\frac{-N}{p}\right)=0$ when $p \mid N$.

Proof. A representation of $N$ by $Q=x^{2}+2 y^{2}+2 z^{2}$ is called primitive if $\operatorname{gcd}(x, y, z)=1$. If $8 n+5$ is square-free, then $C_{4}(n)=R(8 n+5, Q)$ is the number of positive primitive representations of $8 n+5$ by $Q$ since there can be no non-primitive representations. However, in the general case, let $r(n, Q)$ denote the number of primitive representations of $n$ by $Q$. It follows that

$$
C_{4}(n)=R(8 n+5, Q)=\sum_{d^{2} \mid 8 n+5} r\left(\frac{8 n+5}{d^{2}}, Q\right) .
$$

However, by an argument similar to the one given in Theorem 2, it follows that

$$
r\left(\frac{8 n+5}{d^{2}}\right)=\frac{1}{2} h\left(\frac{-32 n-20}{d^{2}}\right) .
$$

The multiplicative formula now follows from the following well known property which expresses $h\left(-N f^{2}\right)$ in terms of $h(-N)$ when $-N$ is a fun- 
damental discriminant $[8,7.28]$ :

$$
h\left(-N f^{2}\right)=h(-N) f \prod_{p \mid f}\left(1-\frac{\left(\frac{-N}{p}\right)}{p}\right) .
$$

However, it is an easy exercise to verify that the above formula generalizes if $-N$ is not square-free. In particular, it follows that if $\operatorname{ord}_{p}(N) \leq 1$, then for every positive integer $i$,

$$
h\left(-N p^{2 i}\right)=h(-N) p^{i}\left(1-\frac{\left(\frac{-N}{p}\right)}{p}\right) .
$$

Therefore by (4)-(6) we find that

$$
\begin{aligned}
C_{4}\left(\frac{N p^{2 k}-5}{8}\right) & =R\left(N p^{2 k}, Q\right)=\sum_{0 \leq i \leq k} \sum_{d^{2} \mid N} r\left(\frac{N p^{2 i}}{d^{2}}, Q\right) \\
& =\frac{1}{2} \sum_{0 \leq i \leq k} \sum_{d^{2} \mid N} h\left(\frac{-N p^{2 i}}{d^{2}}\right) \\
& =\frac{1}{2} \sum_{d^{2} \mid N} h\left(\frac{-N}{d^{2}}\right)+\frac{1}{2} \sum_{1 \leq i \leq k} \sum_{d^{2} \mid N} h\left(\frac{-N p^{2 i}}{d^{2}}\right) \\
& =C_{4}\left(\frac{N-5}{8}\right)+\frac{1}{2} \sum_{1 \leq i \leq k} \sum_{d^{2} \mid N} h\left(\frac{-N}{d^{2}}\right) p^{i}\left(1-\frac{\left(\frac{-N}{p}\right)}{p}\right) \\
& =C_{4}\left(\frac{N-5}{8}\right)+C_{4}\left(\frac{N-5}{8}\right)\left(1-\frac{\left(\frac{-N}{p}\right)}{p}\right) \sum_{1 \leq i \leq k} p^{i} .
\end{aligned}
$$

It is easy to verify that this agrees with the asserted formula.

It is clear that one obtains many congruences for $C_{4}(n)$ from Corollary 2. However, by elementary genus theory, we obtain a different sort of congruence.

COROLlary 3. If $8 n+5$ is a positive square-free integer with $t$ prime divisors, then

$$
C_{4}(n) \equiv 0\left(\bmod 2^{t-1}\right) .
$$

It turns out that it is easy to determine the exact parity of $C_{4}(n)$ directly. The conjugate of a partition $\Lambda$ is the partition whose parts are the column lengths of the Ferrers-Young diagram of $\Lambda$. Since the conjugate partition of a 4-core is also a 4-core, determining those $n$ for which $C_{4}(n)$ is odd reduces to finding all $n$ that admit an odd number of self-conjugate 4-core partitions. Here we compute $\mathrm{sc}_{4}(n)$, the number of self conjugate 4-cores of $n$. This result is also given in [19], and an analogous result is obtained by Garvan, Kim, and Stanton for 5-cores [13, Th.7]. 
THEOREM 3. Let $\mathrm{sc}_{4}(n)$ denote the number of self-conjugate 4-core partitions of $n$. If the factorization of $8 n+5$ into distinct primes $p_{i}$ and $q_{j}$ is

$$
8 n+5=\prod p_{i}^{\alpha_{i}} q_{j}^{\beta_{j}}
$$

where $p_{i} \equiv 1(\bmod 4)$ and $q_{j} \equiv 3(\bmod 4)$, then

$$
\operatorname{sc}_{4}(n)= \begin{cases}0 & \text { if any } \beta_{j} \equiv 1(\bmod 2), \\ \frac{1}{2} \prod\left(\alpha_{i}+1\right) & \text { otherwise }\end{cases}
$$

Proof. We use the bijection given by Garvan, Kim, and Stanton [13] between $t$-cores and solutions to particular quadratic forms. In particular, the number of 4-core partitions of $n$ is equal to the number of vectors $\left(n_{0}, n_{1}, n_{2}, n_{3}\right) \in \mathbb{Z}^{4}$ on the hyperplane $n_{0}+n_{1}+n_{2}+n_{3}=0$ which satisfy

$$
n=2\left(n_{0}^{2}+n_{1}^{2}+n_{2}^{2}+n_{3}^{2}\right)+n_{1}+2 n_{2}+3 n_{3} .
$$

Furthermore, under that bijection, the 4-tuples $\left(n_{0}, n_{1}, n_{2}, n_{3}\right)$ and $\left(-n_{3}\right.$, $\left.-n_{2},-n_{1},-n_{0}\right)$ correspond to conjugate 4 -cores. Therefore, self-conjugate 4 -cores correspond exactly to 4 -tuples of the form $(a, b,-b,-a)$. It follows that the number of self-conjugate 4-cores of $n$ is the number of solutions of

$$
16 n+10=(8 a-3)^{2}+(8 b-1)^{2} .
$$

By congruences, these are the only ways of writing $16 n+10$ as the sum of two squares up to reorderings and sign changes. Thus, the number of 4-core partitions of $n$ is the number of ways to get the factorization $16 n+10=$ $x^{2}+y^{2}=(x+i y)(x-i y)$ up to sign changes on the $x$ and $y$. This number is well known to be 0 if $\operatorname{ord}_{q_{j}}(16 n+10) \equiv 1(\bmod 2)$ for any $q_{j}$ and is equal to the number of positive factors of $8 n+5$ divisible only by primes congruent to $1(\bmod 4)$, otherwise. We divide the divisor function $\prod\left(\alpha_{i}+1\right)$ in half to get the actual number of usable solutions since $8 n+5$ is not a perfect square, and the number of factorizations is counted twice when counting divisors.

Remark 1 . The value $C_{4}(8 n+5)$ is odd if and only if there are an odd number of self-conjugate 4-cores of $n$. In general, $\operatorname{sc}_{4}(n)$ is odd if and only if

$$
8 n+5=\prod p^{\alpha} N^{2}
$$

where $p$ is a prime, $\operatorname{gcd}(p, N)=1, \alpha \equiv 1(\bmod 4)$ and $p \equiv 1(\bmod 4)$.

3. Correspondence between 4-cores and binary quadratic forms. In this section, we construct a correspondence between 4-cores and binary quadratic forms which yields an alternative proof of Theorem 2. We use the combinatorial theory of "abaci" as developed in $[10,21,28]$ to classify 4-cores which leads to a structure theorem where the parts of any 4-core partition are determined. 
Under this correspondence, if $8 n+5$ is square-free with $t$ prime divisors, then the set of 4 -cores of $n$ is a $2^{t-1}$-fold cover of a unique genus in $\mathrm{CL}(-32 n-20)$. In particular, if $8 n+5$ is prime, then the set of 4 -cores of $n$ is naturally an abelian group with odd order, namely the subgroup of squares in $\mathrm{CL}(-32 n-20)$.

Let $\Lambda=\lambda_{1} \geq \ldots \geq \lambda_{s}>0$ be a partition of a positive integer $n$. Then for any positive integer $t \geq 2$, there exists an associated abacus consisting of $s$ beads on $t$ "rods" built in the following manner. For each $1 \leq i \leq s$, define structure numbers

$$
B_{i}=\lambda_{i}-i+s .
$$

Note that the integers $B_{i}$ are strictly decreasing by construction. For each $B_{i}$, there is a unique pair of integers $\left(r_{i}, c_{i}\right)$ for which

$$
B_{i}=t\left(r_{i}-1\right)+c_{i},
$$

and $0 \leq c_{i} \leq t-1$. An abacus for $t$-cores consists of $t$ rods numbered from 0 to $t-1$, and infinitely many rows numbered in increasing order. The abacus for the partition $\Lambda$ consists of one bead for each $B_{i}$ placed in row $r_{i}$ and column $c_{i}$, a position we denote by $\left(r_{i}, c_{i}\right)$.

EXAMPLE 2. If $\Lambda$ denotes the 4 -core partition of 12 given by $\Lambda:=7,4,1$, then the structure numbers are $B_{1}=7-1+3=9, B_{2}=4-2+3=5$, and $B_{3}=1-3+3=1$. The abacus when $t=4$ consists of a single column of beads in positions $(3,1),(2,1)$, and $(1,1)$. Graphically, the abacus for this partition is

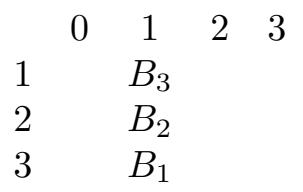

THEOREM 4. Let $\mathfrak{A}$ be an abacus for a partition $\Lambda$, and let $n_{i}$ denote the number of beads in column $i$. Then $\Lambda$ is a t-core partition if and only if for every $0 \leq i \leq t-1$ the $n_{i}$ beads in column $i$ are the beads in positions

$$
(1, i),(2, i), \ldots,\left(n_{i}, i\right) .
$$

In other words, there are no gaps between consecutive beads in any column, and the top bead in every non-empty column is in row 1 .

Therefore, we may let $t$-tuples of non-negative integers $\mathfrak{A}=\left(n_{0}, \ldots, n_{t-1}\right)$ denote the abaci of $t$-cores. As the following lemma shows, these "gapless" abacus positions do not represent $t$-cores uniquely [10] when we allow for parts of size 0 in partitions.

Lemma 1. The two abaci $\mathfrak{A}_{1}=\left(n_{0}, n_{1}, \ldots, n_{t-1}\right)$ and $\mathfrak{A}_{2}=\left(n_{t-1}+\right.$ $\left.1, n_{0}, n_{1}, \ldots, n_{t-2}\right)$ represent the same $t$-core partition. 
Now because of this lemma, it is clear that there are many abaci for any given partition once we allow for abaci where the number of beads exceeds the number of parts in a partition. In such cases, some of the beads will correspond to parts of size zero in the partition.

Since it is our goal to use abaci as labels for all $t$-cores, it is important for us to normalize them properly. Although the following exposition may be generalized to arbitrary $t$-cores, it is not necessary for the development of our main goals. Hence, we restrict our attention to the case where $t=4$.

Every 4-core has a representation by a 4-tuple which in turn by repeated application of Lemma 1 is representable by an abacus of the form $(0, B, C, D)$. The bead in the upper-left corner of such an abacus corresponds to the smallest part of the partition and clearly is one of 1,2 , or 3 since these are the only integers representable by beads in positions $(1,1),(1,2)$, or $(1,3)$. Hence, it is clear that there is a unique abacus of the form $(0, B, C, D)$ for every 4-core, Thus, there is a one-to-one correspondence between the set of abaci of the form $(0, B, C, D)$ and the set of all 4-cores

$$
(0, B, C, D) \leftrightarrow\{\text { all } 4 \text {-core partitions }\}
$$

where $B, C$, and $D$ are non-negative integers. Therefore, we shall always assume that the first column in every abacus contains no beads.

Lemma 2. If $\mathfrak{A}_{1}=(0, B, C, D)$ is a 4-core partition of $n$, then $\mathfrak{A}_{2}=$ $(0, D+1, B, C)$ is a 4-core partition of $n+D+1$.

Thus, by Lemma 2, we find that 4-cores give birth to other 4-cores in a very systematic way.

Definition 2. A 4-core partition of $n$ is called new if it is represented by an abacus of the form $\mathfrak{A}=(0,0, C, D)$.

By Lemma 2, it is easy to see that every 4-core has a unique new core ancestor, and is one of three types. The following definition captures their essential characteristics.

Definition 3 . Let $\mathfrak{A}=(0,0, C, D)$ be a new 4-core partition and let $g$ be a non-negative integer.

I. The Type I generation $g$ descendant of $\mathfrak{A}$ is the 4-core whose abacus is of the form $(0, g, C+g, D+g)$. Denote this 4 -core by $I(g, C, D)$.

II. The Type II generation $g$ descendant of $\mathfrak{A}$ is the 4 -core whose abacus is of the form $(0, D+g+1, g, C+g)$. Denote this 4-core by $I I(g, C, D)$.

III. The Type III generation $g$ descendant of $\mathfrak{A}$ is the 4 -core whose abacus is of the form $(0, C+g+1, D+g+1, g)$. Denote this 4-core by $I I I(g, C, D)$.

We now state the structure theorem that determines the parts of every 4-core partition. 
Theorem 5 (Structure Theorem). Let $\mathfrak{A}=(0,0, C, D)$ be a new 4-core partition and let $g \geq 0$ be a non-negative integer.

I. Define integers $d$ and e by

$$
d:=\min (C, D) \quad \text { and } \quad e:=|C-D| .
$$

If $C \geq D$, then the parts of the 4-core $I(g, C, D)$ are:

$$
\begin{aligned}
& g+2 d+3 e-1, g+2 d+3 e-4, \ldots, g+2 d+2 \quad(\text { e integers }), \\
& g+2 d, g+2 d, g+2 d-2, g+2 d-2, \ldots, g+2, g+2 \quad(d \text { pairs }), \\
& g, g, g, g-1, g-1, g-1, \ldots, 1,1,1 \quad(g \text { triples }) .
\end{aligned}
$$

If $C<D$, then the parts of the 4-core $I(g, C, D)$ are:

$$
\begin{aligned}
& g+2 d+3 e, g+2 d+3 e-3, \ldots, g+2 d+3 \quad \text { (e integers), } \\
& g+2 d, g+2 d, g+2 d-2, g+2 d-2, \ldots, g+2, g+2 \quad(d \text { pairs }), \\
& g, g, g, g-1, g-1, g-1, \ldots, 1,1,1 \quad \text { (g triples). }
\end{aligned}
$$

II. Define integers $d$ and $e$ by

$$
d:=\min (2 C+1,2 D+1) \quad \text { and } \quad e:=|C-D| .
$$

If $C \geq D$, then the parts of the 4-core $I I(g, C, D)$ are:

$g+d+3 e-2, g+d+3 e-5, \ldots, g+d+1 \quad$ (e integers),

$g+d, g+d-1, g+d-2, \ldots, g+1 \quad$ (d consecutive integers),

$g, g, g, g-1, g-1, g-1, \ldots, 1,1,1 \quad$ (g triples).

If $C<D$, then the parts of the 4-core $I I(g, C, D)$ are:

$g+d+3 e, g+d+3 e-3, \ldots, g+d+3$ (e integers),

$g+d, g+d-1, g+d-2, \ldots, g+1 \quad$ (d consecutive integers),

$g, g, g, g-1, g-1, g-1, \ldots, 1,1,1 \quad$ (g triples).

III. Define integers $d$ and $e$ by

$$
d:=\min (C+1, D+1) \quad \text { and } \quad e:=|C-D| .
$$

If $C \geq D$, then the parts of the 4-core $\operatorname{III}(g, C, D)$ are:

$g+2 d+3 e-2, g+2 d+3 e-5, \ldots, g+2 d+1 \quad$ (e integers),

$g+2 d-1, g+2 d-1, g+2 d-3, g+2 d-3, \ldots, g+1, g+1 \quad$ (d pairs),

$g, g, g, g-1, g-1, g-1, \ldots, 1,1,1 \quad$ (g triples).

If $C<D$, then the parts of the 4-core $\operatorname{III}(g, C, D)$ are:

$g+2 d+3 e-1, g+2 d+3 e-4, \ldots, g+2 d+2$ (e integers),

$g+2 d-1, g+2 d-1, g+2 d-3, g+2 d-3, \ldots, g+1, g+1 \quad$ (d pairs),

$g, g, g, g-1, g-1, g-1, \ldots, 1,1,1 \quad$ (g triples). 
Proof. Two key observations and an illustration are first in order.

Observation 1 . The smallest part in a 4-core partition is given by $\lambda_{s}=B_{s}$, the structure number of the upper-left bead in the abacus.

Observation 2. The difference between two consecutive structure numbers $B_{i}$,

$$
B_{i-1}-B_{i}=\left(\lambda_{i-1}-(i-1)+s\right)-\left(\lambda_{i}-i+s\right)=\lambda_{i-1}-\lambda_{i}+1
$$

is one more than the difference between the sizes of two consecutive parts. Consequently, we find that

$$
\lambda_{i-1}=\left(B_{i-1}-B_{i}\right)+\lambda_{i}-1 .
$$

All the parts are obtained iteratively from the structure numbers in the following way. To determine $\lambda_{i-1}$ from $\lambda_{i}$, simply add $B_{i-1}-B_{i}-1$ to $\lambda_{i}$. Fortunately, it is simple to deduce $B_{i-1}-B_{i}$ from the abacus.

The following illustration will be helpful. Consider the 4-core represented by $I(1,2,1)$. By Definition 3 we use the abacus $\mathfrak{A}=(0,1,3,2)$ :

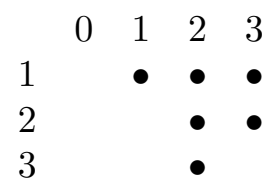

If we replace each of the beads in this abacus by their associated structure numbers, $B_{1}=10, B_{2}=7, B_{3}=6, B_{4}=3, B_{5}=2$, and $B_{6}=1$, we get the following abacus.

$\begin{array}{ccccc} & 0 & 1 & 2 & 3 \\ 1 & & 1 & 2 & 3 \\ 2 & & & 6 & 7 \\ 3 & & & & 10\end{array}$

Since the smallest part is $\lambda_{6}=B_{6}=1$, we may now get the remaining parts inductively: $\lambda_{5}=1, \lambda_{4}=1, \lambda_{3}=3, \lambda_{2}=3$, and $\lambda_{1}=5$.

We now prove the case illustrated by the above example.

$\mathrm{C}$ a s e I (for the structure of $I(g, C, D)$ where $C \geq D)$. I $(g, C, D)$ corresponds by Definition 3 to the abacus of the form $\mathfrak{A}=(0, g, C+g, D+g)$. This abacus consists, from the top down, of up to three distinct sections. The first section consists of $g$ completely filled rows except for column 0 . The second section consists of $d=C$ rows with beads in columns 2 and 3 . Finally, the last section consists of $e=C-D$ rows with beads only in column 2. We shall refer to these three parts as the $g$-block, $d$-block and e-block.

We begin by first examining the $g$-block. If $g \geq 1$, the $g$-block consists of beads in positions $(r, s)$ for $1 \leq r \leq g$ and $1 \leq s \leq 3$. The set of structure 
numbers for these beads is

$$
\begin{aligned}
\left\{z \in \mathbb{Z}^{+}: 1 \leq z \leq 4 g-1 \text { and } z \not \equiv 0(\bmod 4)\right\} \\
\quad=\{1,2,3,5,6,7,9,10,11, \ldots, 4 g-5,4 g-3,4 g-2,4 g-1\} .
\end{aligned}
$$

Since the smallest structure number is 1 , the smallest part size is also 1 . Now we inductively produce the remaining part sizes. By Observation 2, the next few part sizes are given by

$$
\begin{aligned}
& (2-1)+1-1=1, \\
& (3-2)+1-1=1, \\
& (5-3)+1-1=2, \\
& (6-5)+2-1=2,
\end{aligned}
$$

Since the structure number of the three consecutive beads in each row differ by 1 , the three beads represent one part size. Furthermore, since the structure number of beads in positions $(r, 3)$ and $(r+1,1)$ differ by $2=4(r+1-1)+1-(4(r-1)+3)$, consecutive rows of beads represent parts that differ by 1 . Thus, the $g$-block represents the following parts:

$$
g, g, g, g-1, g-1, g-1, \ldots, 1,1,1 \quad \text { ( } g \text { triples }) .
$$

If $g=0$, then the above formulation still holds because no parts are represented.

The analysis of the $d$-block, which consists of $d=\min (C, D)=D$ rows of beads in column 2 and column 3 is similar. Since the two columns are adjacent, the structure numbers represented by each row of beads differ by one. Thus, each row represents a pair of parts of equal size. Since beads in positions $(r, 3)$ and $(r+1,2)$ have the property that their structure numbers differ by $3=4(r+1-1)+2-(4(r-1)+3)$, consecutive rows of the $d$-block represent part sizes that differ by 2 .

Finally, we determine the size represented by the top row of this block by noting that the difference of the structure numbers of the adjoining beads between the $d$-block, $(g+1,2)$, and the $g$-block, $(g, 3)$, is $3=4(g+1-1)+$ $2-(4(g-1)+3)$; thus, the size represented by the first row of this block is $g+3-1=g+2$. Hence, all the parts represented by the $d$-block are:

$$
g+2 d, g+2 d, g+2 d-2, g+2 d-2, \ldots, g+2, g+2 \quad \text { (d pairs). }
$$

If $g=0$ and $d>0$, there is no $g$-block, and the $d$-block bead $(1,2)$ is the bead with the smallest structure number, 2. Thus, 2 is also the smallest part. In this case the $d$-block still satisfies the above formula.

The $e$-block consists of the $e=C-D$ beads in column 2 at the abacus bottom. Because the structure numbers of consecutive beads in a column 
differ by 4 , the $e$-block represents consecutive parts whose sizes differ by 3 . The bead with the smallest structure number in this block is $(g+d+1,2)$. If $g+d>0$, then the bead with the largest structure number not in the $e$-block is $(g+d, 3)$. In this case, the difference between the structure numbers of these two beads is $3=(4(g+d+1-1)+2)-(4(g+d-1)+3)$. Thus, the first $e$-block part is 2 more than the largest non-e-block part, $g+2 d$. Therefore, the $e$-block represents the following parts:

$$
g+2 d+3 e-1, g+2 d+3 e-4, \ldots, g+2 d+5, g+2 d+2 \quad \text { (e integers). }
$$

If $g=d=0$ and $e>0$, then the $e$-block bead $(1,2)$ is the bead with the smallest structure number, 2 . Thus, the above formulation for the $e$-block parts with $d=0$ and $g=0$ still holds. If $d=e=g=0$, then there are no beads and no parts.

The other cases follow in exactly the same way.

Similar structure theorems for $t$-cores when $t \neq 4$ are derived in exactly the same way. The following elementary proposition is the critical link required for defining the correspondence between 4-cores and binary quadratic forms.

Proposition 1. Let $\mathfrak{A}=(0,0, C, D)$ be a new 4-core partition and let $g$ be a non-negative integer.

I. $I(g, C, D)$ is a partition of $n$ where

$8 n+5=(2 C-2 D+2 g)^{2}+(2 C+2 D+2 g+2)^{2}+(2 C-2 D-2 g-1)^{2}$.

II. $I I(g, C, D)$ is a partition of $n$ where

$8 n+5=(2 C-2 D+2 g)^{2}+(2 C+2 D+2 g+3)^{2}+(2 C-2 D-2 g-2)^{2}$.

III. $I I I(g, C, D)$ is a partition of $n$ where

$8 n+5=(2 C-2 D+2 g+1)^{2}+(2 C+2 D+2 g+4)^{2}+(2 C-2 D-2 g-2)^{2}$.

Pr o of. We only prove the case where $C \geq D$ since the remaining proofs are similar.

By the Structure Theorem, we can determine the size of the partition $I(g, C, D)$ by simply adding up the parts given by the Structure Theorem.

$$
\begin{aligned}
8 n+5= & 8\left(3 \sum_{i=1}^{g} i+2 \sum_{i=1}^{D}(g+2 i)+\sum_{i=1}^{C-D}(g+2 D+3 i-1)\right)+5 \\
= & 8\left(\frac{3}{2} g(g+1)+\left(2 g D+2 D^{2}+2 D\right)\right. \\
& \left.+\left(g(C-D)+2 D(C-D)+\frac{1}{2}(C-D)+\frac{3}{2}(C-D)^{2}\right)\right)+5 \\
= & 5+12 g+12 D+8 g D+12 g^{2}+4 C+8 g C-8 D C+12 C^{2}+12 D^{2} .
\end{aligned}
$$

This is exactly the expansion of $(2 C-2 D+2 g)^{2}+(2 C+2 D+2 g+2)^{2}+(2 C-$ $2 D-2 g-1)^{2}$. The remaining 5 cases follow in exactly the same manner. 
Remark 2. Proposition 1 follows easily from the work of Erdmann and Michler in [10]. Proposition 1 shows that to each 4-core partition of $n$ there is a representation of $8 n+5=x^{2}+y^{2}+z^{2}$ as a sum of three squares. By congruence conditions, $x, y$, and $z$ belong to some permutation of the residue classes $1(\bmod 2), 2(\bmod 4)$, and $0(\bmod 4)$. Let

$$
\begin{aligned}
J(8 n+5)=\left\{(x, y, z) \in \mathbb{Z}^{3}: 8 n+5=\right. & x^{2}+y^{2}+z^{2}, \\
& (x, y, z) \equiv( \pm 1,2,0)(\bmod 4)\} .
\end{aligned}
$$

Now fix a map $\psi:\{4$-cores $\} \rightarrow J(8 n+5)$ defined by:

$$
\begin{aligned}
I(g, C, D) & \rightarrow(2 C-2 D-2 g-1,2 C-2 D+2 g, 2 C+2 D+2 g+2), \\
I I(g, C, D) & \rightarrow(2 C+2 D+2 g+3,2 C-2 D+2 g, 2 C-2 D-2 g-2), \\
I I I(g, C, D) & \rightarrow \\
& (2 C-2 D+2 g+1,2 C+2 D+2 g+4,2 C-2 D-2 g-2),
\end{aligned}
$$

where the triples are reordered if necessary so that they belong to $J(8 n+5)$. Let

$$
K(8 n+5)=J(8 n+5) / \sim,
$$

where $(x, y, z) \sim\left(x^{\prime}, y^{\prime}, z^{\prime}\right)$ if they differ by 2 sign changes. For example, $(1,2,4) \sim(1,-2,-4)$.

Proposition 2. The map $\psi$ is a bijection between $\{4$-cores of $n\}$ and $K(8 n+5)$.

P r o o f. We restrict ourselves to establishing the correspondence between type $I I(g, C, D)$ and $(x, y, z) \in K(8 n+5)$ where $|x|=\max (|x|,|y|,|z|)$. Such triples can only be the image of a $I I(g, C, D)$ because it is the only type where the largest entry, in absolute value, in the triple $(x, y, z)$ is odd.

So we simply need to show that all such classes with representative $(x, y, z) \in K(8 n+5)$ are images of a unique $I I(g, C, D)$ partition. So suppose that $x=2 C+2 D+2 g+3$. Then either

or

$$
\left\{\begin{array}{l}
y=2 C-2 D+2 g \\
z+2=2 C-2 D-2 g,
\end{array}\right.
$$

$$
\left\{\begin{array}{l}
z=2 C-2 D+2 g \\
y+2=2 C-2 D-2 g .
\end{array}\right.
$$

The choice is forced by whether $y \equiv x+1(\bmod 4)$ or $z \equiv x+1(\bmod 4)$. Without loss of generality suppose that $y \equiv x+1(\bmod 4)$ and $2 C-2 D-$ $2 g=z+2$. Then solving for $C, D$, and $g$ yields

$$
D=\frac{x-y-3}{4}, \quad g=\frac{y-z-2}{4} \quad \text { and } \quad C=\frac{x+z-1}{4} \text {. }
$$

By construction, these are all integers, and we simply need to show that precisely one of the elements in the class of $(x, y, z)$ in $K(8 n+5)$ has the 
property that $g, C$, and $D$ determined in this manner are non-negative integers. It is easy to see that $x$ must be positive. Let $(x, y, z)$ be one of the elements in the class with this property. The only two possible elements are $(x, y, z)$ and $(x,-y,-z)$. Furthermore, $D, C \geq 0$ since $x$ is maximal. Precisely one of these triples has the desired property that $g \geq 0$ also.

The second case is slightly messier, and in a similar way we obtain a bijection between the set of type I's and III's and the remaining classes of $K(8 n+5)$.

By Gauss [14, Art. 278], for each representation of $8 n+5$ as a sum of three squares, there corresponds a binary quadratic form of discriminant $-32 n-20$. This correspondence is invariant under a pair of simultaneous sign changes on a solution to the representation of $8 n+5$ as a sum of three squares.

Explicitly, his correspondence is as follows: for $(x, y, z) \in J(8 n+5)$ let $\left(m_{0}, m_{1}, m_{2}, n_{0}, n_{1}, n_{2}\right)$ be an integral solution to

$$
x=m_{1} n_{2}-m_{2} n_{1}, \quad y=m_{2} n_{0}-m_{0} n_{2}, \quad z=m_{0} n_{1}-m_{1} n_{0}
$$

(a solution is guaranteed by [14, Art. 279]). Then

$$
\left(m_{0} u+n_{0} v\right)^{2}+\left(m_{1} u+n_{1} v\right)^{2}+\left(m_{2} u+n_{2} v\right)^{2}
$$

is a form in CL $(-32 n-20)$. Moreover, this map is independent of $\left(m_{0}, m_{1}\right.$, $\left.m_{2}, n_{0}, n_{1}, n_{2}\right)$. Therefore, there is a map $\phi:\{4$-cores of $n\} \rightarrow \mathrm{CL}(-32 n-$ $20)$, when $8 n+5$ is square-free, formed by composing $\psi,(8)$, and (9). It is given by

$\phi: \Lambda \rightarrow \mathfrak{A} \rightarrow(x, y, z) \rightarrow\left(m_{0}, m_{1}, m_{2}, n_{0}, n_{1}, n_{2}\right) \rightarrow$ binary quadratic form.

Remark 3. Here are some important observations which will be used in the sequel.

(i) Changing the sign on one element of $(x, y, z) \in J(8 n+5)$ to say, $(-x, y, z)$ changes the solution for $(8)$ to $\left(m_{0},-m_{1},-m_{2},-n_{0}, n_{1}, n_{2}\right)$ and the corresponding binary quadratic form (9) becomes

$$
\left(m_{0} u-n_{0} v\right)^{2}+\left(-m_{1} u+n_{1} v\right)^{2}+\left(-m_{2} u+n_{2} v\right)^{2} .
$$

This is the inverse to the quadratic form (9) associated with $(x, y, z)$. Thus, Gauss' map remains invariant over pairs of sign changes, and is thus well defined on $K(8 n+5)$.

(ii) If one of $x, y$, or $z$ is zero, the associated binary quadratic form is the principal form. For instance suppose that $z=0$. Then a solution to (8) is $m_{0}=0, n_{0}=-y, m_{1}=x, n_{1}=0, m_{2}=0$, and $n_{2}=1$. Substituting into (9), we get

$$
(-y u)^{2}+(x u)^{2}+v^{2}=\left(x^{2}+y^{2}\right) u^{2}+v^{2}=(8 n+5) u^{2}+v^{2},
$$

which is a representative for the principal class. 
Before proceeding, we prove the following conjugation identities which are useful in the sequel. We shall let $\mathfrak{A}_{1} \sim \mathfrak{A}_{2}$ signify that $\mathfrak{A}_{1}$ and $\mathfrak{A}_{2}$ are conjugate partitions.

Proposition 3. The following pairs of partitions are conjugate 4-core partitions.

(i) If $D \geq C$, then

$$
\begin{aligned}
I(g, C, D) & \sim I(D-C, C, C+g), \\
I I(g, C, D) & \sim I I(D-C, C, C+g) .
\end{aligned}
$$

(ii) If $D<C$, then

$$
\begin{aligned}
I I(g, C, D) & \sim I I(C-D-1, D+g+1, D), \\
I I I(g, C, D) & \sim I I I(C-D-1, D+g+1, D) .
\end{aligned}
$$

(iii) If $D<C$, then

$$
I(g, C, D) \sim I I I(C-D-1, D, D+g) .
$$

(iv) If $D \geq C$, then

$$
I I I(g, C, D) \sim I(D-C, g+C+1, C) .
$$

Proof. Here we prove the case where $I(g, C, D) \sim I(D-C, C, C+g)$ and $D \geq C$. By Theorem 3, the partition associated with $I(g, C, D)$ is

$$
\begin{aligned}
& g+3 D-C, g+3 D-C-3, \ldots, g+2 C+3 \quad(D-C \text { integers }), \\
& g+2 C, g+2 C, g+2 C-2, g+2 C-2, \ldots, g+2, g+2 \quad(C \text { pairs }), \\
& g, g, g, g-1, g-1, g-1, \ldots, 1,1,1 \quad(g \text { triples }) .
\end{aligned}
$$

Conjugation of a Ferrers-Young diagram switches rows and columns, so it will be enough to show that the column sizes of the above partition correspond to the row sizes given by the Structure Theorem for $I(D-C, C, C+g)$.

We examine the columns of the Ferrers-Young diagram for $I(g, C, D)$ from right to left. The part of the top $D-C$ rows in the original partition that sticks out over the bottom two blocks of rows will have column sizes 1 through $D-C$ in groups of three's. The middle $2 C$ rows of doubles increasing by 2's give $2 C$ columns whose part sizes come in pairs starting at $D-C+2$ and ending at $D+C$. Finally, the last $3 g$ rows of triples give rise to the final $g$ columns. These columns differ by 3's and start in size at 3 greater than the previous column, and give the column sizes $D+C+3, D+C+6, \ldots, D+$ $C+3 g$. These are exactly the parts given by the Structure Theorem for $I(D-C, C, C+g)$. The other cases follow in a similar fashion.

Note that conjugating a partition of $n$ amounts to changing the sign of one element of the associated representation of $8 n+5$ by a sum of three 
squares. For example, in the first conjugation identity $I(g, C, D) \sim I(D-$ $C, C, C+g)$, the associated triples, before reordering, are

$$
(2 C-2 D-2 g-1,2 C-2 D+2 g, 2 C+2 D+2 g+2)
$$

and

$$
(2 C-2 D-2 g-1,-2 C+2 D-2 g, 2 C+2 D+2 g+2) .
$$

Thus, self-conjugate 4-core partitions of $n$ are exactly those whose associated triples have a 0 . Therefore, the number of self-conjugate 4-core partitions of $n$ is exactly the number of ways to write $8 n+5$ as the sum of the squares of two positive integers, disregarding order. This gives a second proof of Theorem 3.

Remark 4. By Remark 3(i), conjugate 4-cores correspond to inverse classes of quadratic forms, and by Remark 3(ii), self conjugate 4-cores map to the class containing the principal form. The reader should be aware that not all order 2 elements have to be images of self-conjugate partitions (see Example 4).

With these preliminaries, we prove:

THEOREM 6. If $8 n+5$ is a positive square-free integer with $t$ prime divisors, then the image of $\phi$ is a unique genus of binary quadratic forms with discriminant $-32 n-20$, and every equivalence class of forms in this genus is the image of $2^{t-1}$ many different 4 -cores of $n$.

Proof. It is well known that $|\mathrm{CL}(-32 n-20)|=2^{t} k$ where $k$ is the number of classes per genus, and $2^{t}$ is the number of genera in CL $(-32 n-$ $20)$. Fix $f_{1}, \ldots, f_{k}$ to be representatives for the $k$ classes in the unique genus of $\operatorname{CL}(-32 n-20)$ that $\phi$ maps onto. We will say that $(x, y, z)$ and $f_{i}$ are represented by $\left(m_{0}, m_{1}, m_{2}, n_{0}, n_{1}, n_{2}\right)$ if

$$
x=m_{1} n_{2}-m_{2} n_{1}, \quad y=m_{2} n_{0}-m_{0} n_{2}, \quad z=m_{0} n_{1}-m_{1} n_{0},
$$

and

$$
\left(m_{0} u+n_{0} v\right)^{2}+\left(m_{1} u+n_{1} v\right)^{2}+\left(m_{2} u+n_{2} v\right)^{2}=f_{i} .
$$

Let $\mathfrak{M}$ denote the set of all such tuples that represent some pair $(x, y, z)$ and $f_{i}$. By Gauss [14, Art. 291], $|\mathfrak{M}|=3 \cdot 2^{t+3} k$, and each $f_{i}$ is representable by $3 \cdot 2^{t+3}$ members of $\mathfrak{M}$.

However, for each $f_{i}$, there are $2^{t+2}$ elements in $\mathfrak{M}$ for which $(x, y, z)$ is in $J(8 n+5)$. Moreover, elements of the form $\left(m_{0}, n_{0}, m_{1}, n_{1}, m_{2}, n_{2}\right)$ and $\left(-m_{0},-n_{0},-m_{1},-n_{1},-m_{2},-n_{2}\right)$ map to the same form. Since there are no other such relations, we find that there are $2^{t+1}$ elements in $\mathfrak{M}$ mapping to each $f_{i}$ for which the triple $(x, y, z)$ is in $J(8 n+5)$. Since $8 n+5$ is square-free, each class in $K(8 n+5)$ corresponds to four distinct triples. Therefore each 
element in $K(8 n+5)$ has $2^{t-1}$ preimages. Consequently, the set of 4-cores of $n$ forms a $2^{t-1}$-fold cover of this genus of forms.

Recall that conjugate partitions have associated triples that differ by a sign on one element. By Remark 4, these triples are mapped to binary quadratic forms that are inverse to each other in CL $(-32 n-20)$. Furthermore, self-conjugate 4-cores of $n$ correspond to the identity element in $\mathrm{CL}(-32 n-20)$. As an immediate corollary we obtain:

COROllary 4. If $8 n+5$ is a positive square-free integer with $t$ prime divisors all congruent to $1(\bmod 4)$, then the set of 4 -cores of $n$ is a $2^{t-1}$ fold cover of $\mathrm{CL}^{2}(-32 n-20)$. In particular, if $8 n+5$ is prime, then the set of 4-core partitions of $n$ forms an abelian group with odd order that is isomorphic to $\mathrm{CL}^{2}(-32 n-20)$.

Proof. By Theorem 6, the 4-cores of $n$ form a $2^{t-1}$-fold cover of one genus in $\mathrm{CL}(-32 n-20)$. By Theorem 3 , this set has exactly $2^{t-1}$ selfconjugate partitions. Since these partitions map to the class of the principal form, it follows that 4-cores of $n$ map to the principal genus. By Gauss [8, Theorem 3.15], this genus is the subgroup of squares $\mathrm{CL}^{2}(-32 n-20)$.

In the case where $8 n+5$ is prime, this map is a bijection between 4-core partitions of $n$ and the subgroup $\mathrm{CL}^{2}(-32 n-20)$. These 4-cores inherit a group structure where the unique self-conjugate partition is the identity, and conjugate partitions correspond to inverses in the class group. Furthermore, since $h(-32 n-20)=2 \cdot C_{4}(n)=$ twice an odd by Theorems 2 and 3 , the set of 4 -cores of $n$ corresponds to the odd part of the class group CL $(-32 n-20)$ which is $\mathrm{CL}^{2}(-32 n-20)$.

ExAmple 3 . Consider $n=22$. In this case $8 n+5=181$ is prime, $h(-4 \cdot 181)=10$, and $\mathrm{CL}(-4 \cdot 181) \cong \mathbb{Z} / 10 \mathbb{Z}$. By Corollary 4 the set of 4 -core partitions of 22 is isomorphic to $\mathrm{CL}^{2}(-724) \cong \mathbb{Z} / 5 \mathbb{Z}$. The complete correspondence here is:

\begin{tabular}{cccc}
\hline Partition & Type & Binary quadratic form & Element \\
\hline $8,5,2,2,2,1,1,1$ & $I(2,0,2)$ & $u^{2}+181 v^{2}$ & identity \\
$8,5,3,3,1,1,1$ & $I(1,3,1)$ & $5 u^{2}+4 u v+37 v^{2}$ & $\gamma$ \\
$7,4,4,2,2,1,1,1$ & $I I I(1,1,2)$ & $5 u^{2}-4 u v+37 v^{2}$ & $\gamma^{4}$ \\
$10,7,4,1$ & $I I(0,0,3)$ & $13 u^{2}+2 u v+14 v^{2}$ & $\gamma^{3}$ \\
$4,3,3,3,2,2,2,1,1,1$ & $I I(3,0,0)$ & $13 u^{2}-2 u v+14 v^{2}$ & $\gamma^{2}$ \\
\hline
\end{tabular}

The correspondence between partitions and the abacus positions is given by Theorem 5. For instance, the partition $8,5,2,2,2,1,1,1$ contains the segment $2,2,2,1,1,1$ from which we deduce that the generation number is $g=2$. Since the remaining parts contain no pairs or consecutive parts, it 
follows that $d=0$. Since there are $e$ parts with consecutive differences of 3 , it follows that $e=|C-D|=2$ and that this partition is a type $I$ partition where $C<D$. Since in this case $d=\min (C, D)=0$, it follows that this partition is a type $I(2,0,2)$ partition.

By $(7), I(2,0,2)$ maps to the 3 -tuple $(-9,10,0)$ which satisfies

$$
(-9)^{2}+10^{2}+0^{2}=181 \text {. }
$$

As a solution to (8)

$$
-9=m_{1} n_{2}-m_{2} n_{1}, \quad 10=m_{2} n_{0}-m_{0} n_{2}, \quad 0=m_{0} n_{1}-m_{1} n_{0},
$$

we find

$$
m_{0}=10, n_{0}=0, \quad m_{1}=9, n_{1}=0, \quad m_{2}=0, n_{2}=-1 .
$$

By substituting this into (9), we obtain

$$
(10 u)^{2}+(9 u)^{2}+(-v)^{2}=181 u^{2}+v^{2},
$$

the principal form.

The second partition $8,5,3,3,1,1,1$ corresponds to the abacus $I(1,3,1)$. By map (7), this abacus corresponds to the triple (1, 6, 12), and Gauss' method gives the quadratic form $5 u^{2}+4 u v+37 v^{2}$. Call the class of this form $\gamma$.

The conjugate to the second partition, 7, 4, 4, 2, 2, 1, 1, 1 maps first to the triple $(1,-6,12)$ which by Remark 3(i) corresponds to the inverse binary quadratic form $5 u^{2}-4 u v+37 v^{2}$. Since the order of $\mathrm{CL}^{2}(-32 n-20)$ is 5 , we find that $5 u^{2}-4 u v+37 v^{2}=\gamma^{4}$. Similarly the fourth partition $10,7,4,1$ corresponds to the binary quadratic form $13 u^{2}+2 u v+14 v^{2}$, and the last partition, 4, 3, 3, 3, 2, 2, 2, 1, 1, 1 corresponds to the form $13 u^{2}-2 u v+14 v^{2}$.

To obtain $\gamma^{2}$, apply the composition law to $\left(5 u^{2}+4 u v+37 v^{2}\right) \cdot\left(5 u^{2}+\right.$ $\left.4 u v+37 v^{2}\right)$. First use the proper transform $(u, v) \rightarrow(-v, u)$ on one of the $\gamma$ 's so that the gcd condition in the Composition Law holds. We find that the unique integer $B(\bmod 2 \cdot 5 \cdot 37)$ satisfying the congruence conditions of the Composition Law is $B=144$, and so the composite form is

$$
37 \cdot 5 \cdot u^{2}+144 u v+\frac{144^{2}+724}{4 \cdot 37 \cdot 5} v^{2}=185 u^{2}+144 u v+29 v^{2} .
$$

This forms reduces to $13 u^{2}-2 u v+14 v^{2}$. Thus $\gamma^{2}$ is equivalent to the image of the partition $4,3,3,3,2,2,2,1,1,1$.

EXAMPLE 4 . Here consider the case where $n=20$ and $8 n+5=165=$ $3 \cdot 5 \cdot 11$. Then by Corollary 4 the set of 4 -core partitions will give a $2^{3-1}=4$ fold cover of a single genus. In this case the genus contains the single class whose representative can be taken to be $6 u^{2}+6 u v+29 v^{2}$. The complete correspondence here is: 


\begin{tabular}{ccc}
\hline Partition & Type & Binary quadratic form \\
\hline $8,5,2,2,1,1,1$ & $I I I(1,0,2)$ & $6 u^{2}+6 u v+29 v^{2}$ \\
$7,4,2,2,2,1,1,1$ & $I(2,2,0)$ & $6 u^{2}+6 u v+29 v^{2}$ \\
$9,6,3,1,1$ & $I I I(0,3,0)$ & $6 u^{2}+6 u v+29 v^{2}$ \\
$5,3,3,2,2,2,1,1,1$ & $I I I(2,1,0)$ & $6 u^{2}+6 u v+29 v^{2}$ \\
\hline
\end{tabular}

Note that the form $6 u^{2}+6 u v+29 v^{2}$ has order 2 in CL $(-660)$ which is consistent with the fact that conjugate partitions correspond to inverses in the group.

4. Concluding remarks. By Theorem 6 , it is easy to see that there is an obvious notion of equivalence which can be applied to 4-core partitions and also to the $r$-defect zero unipotent characters of suitable $\operatorname{GL}(n, q)$ that they label.

Definition 4. Two 4-core partitions of $n$ are equivalent if they correspond to equivalent binary quadratic forms.

With this definition, we obtain the following trivial corollary to Theorem 4.

COROllary 5. If $8 n+5$ is a positive square-free integer with $t$ prime divisors, then there are $k$ equivalence classes of 4-core partitions, each containing $2^{t-1}$-cores of $n$, where $k$ is the number of equivalence classes per genus in $\mathrm{CL}(-32 n-20)$.

It will be interesting to see if this notion of equivalence is meaningful combinatorially or representation theoretically. For instance, the congruences in Corollary 3 are easily seen to follow from Corollary 5. However, is there a natural combinatorial crank that divides the set of 4 -cores of $n$ into equinumerous classes of size $2^{t-1}$ ? If so, then what features, if any, does it share with the ranks and cranks investigated by Andrews, Atkin, Dyson, Garvan, Kim, Stanton, and Swinnerton-Dyer?

In the other direction, can one use these ideas to prove non-trivial theorems about class numbers? It is certainly of interest to see whether one can prove non-trivial theorems about class numbers using the combinatorial properties of 4-core partitions and representation theory.

At this point, it is true that some elementary observations yield interesting, although trivial, tidbits about class numbers. Although the works of Goldfeld, Gross, Oesterlé, and Zagier solve Gauss' class number problem for negative discriminants, it is still of interest to obtain lower bounds for class numbers by other means. For instance, consider the class number 2 problem. By genus theory, it is clear that the only positive square-free integers $8 n+5$ for which $h(-32 n-20)=2$ are primes. By Theorem 5 , it is clear that 3 is a very special integer which defines many symmetric properties of 4 -cores. In 
particular, there should be some nice properties that 4-core partitions satisfy under the 3 permutations of the parameters $g, C$, and $D$ when two of them are equal. These are the 4 -cores of the form $I(x, y, y), I(y, x, y), I(y, y, x)$, $I I(x, y, y), I I(y, x, y), I I(y, y, x), I I I(x, y, y), I I I(y, x, y)$, and $I I I(y, y, x)$ where $x$ and $y$ are non-negative integers.

In particular, consider the following two cases. By Proposition 1, we find that there is a $\operatorname{III}(y, x, y)$ partition of $n$ if and only if

$$
8 n+5=3(2 x+1)^{2}+2(4 y+1)^{2},
$$

and that there is a $I(y, y, x)$ partition of $n$ if and only if

$$
8 n+5=3(2 x+1)^{2}+2(4 y+1)^{2} .
$$

It is well known that every prime $p \equiv 5(\bmod 24)$ is represented by precisely one of the above two forms. However, by Proposition 2 the only self conjugate 4-core partition of the form $I(y, y, x)$ or $I I I(y, x, y)$ is $I(0,0,0)$, which is a partition of 0 . Hence, if $n$ is a positive integer for which $8 n+5 \equiv 5(\bmod 24)$ is prime, then, by Theorem $2, C_{4}(n) \geq 3$. Consequently, by Theorem 1 , if $5<$ $p \equiv 5(\bmod 24)$ is prime, then $h(-4 p) \geq 6$. In particular, we find that $p=5$ is the only prime $p \equiv 5(\bmod 24)$ for which $h(-4 p)=2$. Unfortunately, the same type of elementary arguments fail for primes $p \equiv 13(\bmod 24)$.

Along similar lines, one can find multitudes of elementary identities which force class numbers to be large. For instance, it is easy to verify by Proposition 1 that $I(g, 6,6), I(0,6,6+g), I(g+1,7,2), I I I(4,2, g+$ $3), I I(g, 7,4), I I(2, g+5,4), I(g+2,0,6)$, and $I(6,0, g+2)$ are all 4 -core partitions of $N(g)=\frac{3}{2} g^{2}+\frac{27}{2} g+84$. Moreover, by Proposition 2 , if $g \geq 4$, then these are all distinct partitions. Therefore, if $g \geq 4$ and $N(g)$ is square-free, then by Theorem 1 we find that $h(-32 N(g)-20) \geq 16$.

It will also be of interest to see if these combinatorial interepretations of class numbers will shed light on the Cohen-Lenstra heuristics [7] describing the typical structure of class groups. For instance, will these observations lead to results regarding the probability that an odd prime $p$ divides the class number $h(-32 n-20)$ ?

Acknowledgements. We thank Michael Hirschhorn, Jorn Olsson, David Penniston, and James Sellers for their helpful comments during the preparation of this paper.

Appendix. The connection between 4-core partitions and class numbers is fairly intriguing from the point of view of special values of $L$-functions, weight $\frac{3}{2}$ modular forms, and elliptic curves. Since it is only our intent to draw some analogies, we will be fairly brief and refer to the standard texts and papers for more details. 
If $D=-32 n-20$ is a negative fundamental discriminant, then define $L_{D}(s)$ by

$$
L_{D}(s):=\sum_{n=1}^{\infty} \chi_{D}(n) n^{-s} .
$$

Then Dirichlet's class number formula [8] and Theorem 1 imply that

$$
L_{D}(1)=\frac{4 \pi C_{4}(n)}{\omega(D) \sqrt{|D|}}
$$

where $\omega(D)$ is the number of roots of unity in $\mathbb{Q}(\sqrt{D})$. If $\eta(z):=$ $x^{1 / 24} \prod_{n=1}^{\infty}\left(1-x^{n}\right)$ is Dedekind's eta-function when $x:=e^{2 \pi i z}$, then

$$
\sum_{n=0}^{\infty} C_{4}(n) x^{8 n+5}=\frac{\eta^{4}(32 z)}{\eta(8 z)}
$$

is a weight $\frac{3}{2}$ modular form [34] whose Fourier coefficients are $C_{4}(n)$. Hence Theorem 1 implies that these Fourier coefficients count the number of $r$ defect zero unipotent characters of suitable finite general linear groups, a "local-global" type statistic.

Consider a similar situation for elliptic curves. If $E$ given by

$$
E: \quad y^{2}=x^{3}+A x+B
$$

is an elliptic curve over $\mathbb{Q}$, then let $L(E, s)$ denote its Hasse-Weil $L$-function.

If $D$ is a square-free integer, then the $D$-quadratic twist of $E$, denoted by $E(D)$, is the curve given by

$$
E(D): \quad y^{2}=x^{3}+A D^{2} x+B D^{3} .
$$

Let $\Delta(D)$ be the discriminant of $E(D)$ and let $E_{\text {tor }}(D)$ denote the torsion subgroup of $E(D)$. Furthermore, let $H(D)$ denote the Tate-Shafarevich group of $E(D)$; this group measures the obstruction of the "local-global" principle for elliptic curves. It relates the behavior of $E$ over $\mathbb{Q}$ to the structure of $E$ over the $p$-adic fields where the theory is simpler. In the special case where $E(D)$ has rank zero, the conjectures of Birch and Swinnerton-Dyer imply that

$$
L(E(D), 1)=\frac{|\amalg(D)|}{\left|E_{\mathrm{tor}}(D)\right|} \pi_{\infty} \prod \pi_{p}
$$

where the product is over primes of bad reduction. For complete definitions see [35].

What is interesting is that one component of the special values of the Dirichlet and Hasse-Weil $L$-functions at $s=1$ in (10) and (11) measures "local-global" statistics. The analogy is even a little stronger in the sense that for many elliptic curves $E$ there exists a weight $\frac{3}{2}$ modular form [36] 
$f(z)=\sum_{n=1}^{\infty} A(n) x^{n}$, by Shimura-Waldspurger theory, for many squarefree integers $D$, assuming the conjectures of Birch and Swinnerton-Dyer have the property that $A(D)= \pm \sqrt{|\amalg(D)|}$. These ideas connecting partitions to $\amalg(D)$ have already led to examples of rank zero elliptic curves [31, $32]$. Therefore, in both settings there are modular forms of weight $\frac{3}{2}$ whose Fourier coefficients contain the relevant "local-global" data.

\section{References}

[1] G. Andrews, The Theory of Partitions, Addison-Wesley, 1976.

[2] G. Andrews, C. Bessenrodt and J. Olsson, Partition identities and labels for some modular characters, Trans. Amer. Math. Soc. 344 (1994), 597-615.

[3] G. Andrews and F. Garvan, Dyson's crank of a partition, Bull. Amer. Math. Soc. 18 (1988), 167-171.

[4] A. O. L. Atkin and P. Swinnerton-Dyer, Some properties of partitions, Proc. London Math. Soc. (3) 4 (1954), 84-106.

[5] Z. Borevich and I. Shafarevich, Number Theory, Academic Press, New York, 1966.

[6] R. Brauer, Representations of Finite Simple Groups, Lecture Notes on Modern Math. 1, Wiley, New York, 1963, 133-175.

[7] H. Cohen and H. Lenstra, Heuristics on class groups of number fields, in: Lecture Notes in Math. 1068, Springer, New York, 1984, 33-61.

[8] D. Cox, Primes of the Form $x^{2}+n y^{2}$, Wiley, New York, 1989.

[9] F. Dyson, Some guesses in the theory of partitions, Eureka (Cambridge) 8 (1944), $10-15$.

[10] K. Erdmann and G. Michler, Blocks for symmetric groups and their covering groups and quadratic forms, Beitr. Algebra Geom. 37 (1996), 103-118.

[11] P. Fong and B. Srinivasan, The blocks of finite general linear groups and unitary groups, Invent. Math. 69 (1982), 109-153.

[12] F. Garvan, Some congruence properties for partitions that are $p$-cores, Proc. London Math. Soc. 66 (1993), 449-478.

[13] F. Garvan, D. Kim and D. Stanton, Cranks and t-cores, Invent. Math. 101 (1990), 1-17.

[14] C. F. Gauss, Disquisitiones Arithmeticae, transl. A. A. Clarke, Yale Univ. Press, 1966.

[15] D. Goldfeld, The class number of quadratic fields and the Birch and SwinnertonDyer Conjecture, Ann. Scuola Norm. Sup. Pisa 3 (1976), 623-663.

[16] A. Granville and K. Ono, Defect zero p-blocks for finite simple groups, Trans. Amer. Math. Soc. 348 (1996), 331-347.

[17] B. Gross et D. Zagier, Points de Heegner et derivées de fonctions L, C. R. Acad. Sci. Paris 297 (1983), 85-87.

[18] M. Hirschhorn and J. Sellers, Some amazing facts about 4-cores, J. Number Theory 60 (1996), 51-69.

[19] —, - Two congruences involving 4-cores, Electron. J. Combin. 3 (2) (1996).

[20] M. Isaacs, Character Theory of Finite Simple Groups, Academic Press, New York, 1976 . 
[21] G. James and A. Kerber, The Representation Theory of the Symmetric Group, Addison-Wesley, Reading, 1979.

[22] B. Jones, The Arithmetic Theory of Quadratic Forms, Carus Math. Monographs 10, Math. Assoc. Amer., Wiley, 1950.

[23] I. Kiming, A note on a theorem of A. Granville and K. Ono, J. Number Theory 60 (1996), 97-102.

[24] - On the number of p-spin blocks of defect zero of covering groups of symmetric groups, preprint.

[25] A. Klyachko, Modular forms and representations of symmetric groups, integral lattices and finite linear groups, Zap. Nauchn. Sem. Leningrad. Otdel. Mat. Inst. Steklov. (LOMI) 116 (1982), 74-85 (in Russian).

[26] N. Koblitz, Introduction to Elliptic Curves and Modular Forms, Springer, New York, 1984.

[27] J. Oester lé, Nombre de classes des corps quadratiques imaginaires, Sém. Bourbaki, Astérisque 121-122 (1985), 309-323.

[28] J. Olsson, Combinatorics and representations of finite groups, Univ. Essen Lect. Notes 20, 1993.

[29] K. Ono, On the positivity of the number of t-core partitions, Acta Arith. 66 (1994), 221-228.

[30] - A note on the number of t-core partitions, Rocky Mountain J. Math. 25 (1995), 1165-1169.

[31] - Rank zero quadratic twists of modular elliptic curves, Compositio Math. 104 (1996), 293-304.

[32] - Twists of elliptic curves, ibid., to appear.

[33] G. de Robinson, Representation Theory of the Symmetric Group, Toronto Univ. Press, 1961.

[34] G. Shimura, On modular forms of half-integral weight, Ann. of Math. 97 (1973), 440-481.

[35] J. Silverman, The Arithmetic of Elliptic Curves, Springer, New York, 1986.

[36] J.-L. Waldspurger, Sur les coefficients de Fourier des formes modulaires de poids demi-entier, J. Math. Pures Appl. 60 (1981), 375-484.

School of Mathematics

Institute for Advanced Study

Princeton, New Jersey 08540

U.S.A.

E-mail: ono@math.ias.edu
Department of Mathematics

The Pennsylvania State University University Park, Pennsylvania 16802

U.S.A.

E-mail: sze@math.psu.edu 